\title{
Las características de los Dinki’s: factores de hedonismo y utilitarismo influyentes en el comportamiento de consumo
}

\section{The characteristics of the Dinki's: hedonism and utilitarianism factors influencing consumption behavior}

\author{
SÁNCHEZ-FIGUEROA, Cruz Osbaldo†* \\ Universidad de Colima, Escuela de Mercadotecnia. Avenida Universidad 333, Las Víboras, 28040 Colima, Col.
}

ID $1^{\mathrm{er}}$ Autor: Cruz Osbaldo, Sánchez-Figueroa / ORC ID: 0000-0002-1462-3132

DOI: $10.35429 / J O C S .2019 .20 .6 .7 .14$

Recibido: 19 de Julio, 2019; Aceptado 25 de Septiembre, 2019

\begin{abstract}
Resumen
Objetivo. El propósito de la siguiente investigación es determinar las características hedonistas y utilitaristas de los Dinki's y la relación con el comportamiento de consumo. Metodología. El diseño metodológico fue cuantitativo, exploratorio y descriptivo estudiando el estilo de vida Dinki's, a partir de el instrumento se estudio el grado de hedonismo y utilitarismo, realizado a 224 individuos con las características de Dinkis (Doble ingreso, no hijos), en los municipios de Colima y Villa de Álvarez. El instrumento consistió en una encuesta con escala diferencial semántico, la cual evalúa dos adjetivos opuestos para identificar el Hedonismo y Utilitarismo (Batra \& Ahtola, 1991) compuesto por cinco afirmaciones con cuatro ítems para el Hedonismo y cuatro ítems para el Utilitarismo. Se analizó la validez para determinar su consistencia utilizando el programa estadistíco SPSS, la cual se consideró adecuada, la escala tuvo un alfa de Cronbach de $\alpha=0.932$. Contribución. Se propone un modelo que permita mostrar la relación de las variables, así como los datos obtenidos que determinan las características hedonistas o utilitaristas y su relación con su comportamiento de compra, utilizando un Analisis de Componentes Principales y una regresión.
\end{abstract}

Dink's, Hedonismo, Utilitarismo

\begin{abstract}
Objective The purpose of the following investigation is to determine the hedonistic and utilitarian characteristics of the Dinki and the relationship with the consumption behavior. Methodology The methodological design was quantitative, exploratory and descriptive studying the Dinki's lifestyle, from the instrument the degree of hedonism and utilitarianism was studied, performed on 224 individuals with the characteristics of Dinkis (Double income, no children), in the municipalities of Colima and Villa de Álvarez. The instrument consisted of a survey with a semantic differential scale, which evaluates two opposite adjectives to identify hedonism and utilitarianism (Batra and Ahtola, 1991) composed of five statements with four items for hedonism and four items for utilitarianism. The validity was analyzed to determine its consistency using the SPSS statistical program, the quality was considered adequate, the scale had a Cronbach's alpha of $\alpha=0.932$. Contribution. A model is proposed to show the relationship of the variables, as well as the data obtained that determine the hedonistic or utilitarian characteristics and their relationship with their purchasing behavior, using a Principal Components Analysis and a regression.
\end{abstract}

Dink's, Hedonism, Utilitarianism

Citación: SÁNCHEZ-FIGUEROA, Cruz Osbaldo. Las características de los Dinki's: factores de hedonismo y utilitarismo influyentes en el comportamiento de consumo. Revista de Sociología Contemporánea. 2019. 6-20: 7-14.

\footnotetext{
* Correspondencia al Autor (Correo electrónico: cruzfigueroa@ucol.mx)

$\dagger$ Investigador contribuyendo como primer autor.
} 


\section{Introducción}

La sociedad ha evolucionado y con ello la dinámica de las familias, su estructura, composición y el número de integrantes, lo que ha traído a las nuevas generaciones a pensar en factores que solo generan individualismo como la nulidad del matrimonio, la compra de artículos personales sin opinión de los familiares, hasta las nuevas tendencias de la procreación de hijos.

Con lo anterior ha surgido una tendencia dirigida al no compromiso de educar, cuidar a un hijo, llamada Dinki compuesta por personas que viven en una edad de 25 a 39 años que se encuentran en una situación conyugal, con la firme convicción de no tener un integrante más en la familia. Este modelo de familia llamado así por algunos personajes, es sin duda una de las tendencias que ha recobrado auge para el mundo del marketing, puesto que este tipo de evolución ha sido desde los años atrás con la revolución del movimiento llamado Childless impulsado por su precursora Laura Carroll (2000) y con ello se da origen a otros términos estudiados y analizados desde su funcionalidad como lo es también el Childfree puesto que con ello los dinkis suman fuerzas e ideologías para la conformación de núcleos familiares denominados Dinkis.

En México este estilo de vida está cada día en crecimiento actualmente a través del Instituto de Investigaciones Sociales con la revista digital Datos Diagnósticos Tendencias (DDT) hizo una publicación en octubre 2016 edición 47, se muestra en la siguiente imagen sobre los 11 tipos de familia en México (López Romo, 2016). Con este aporte que la brinda López (2016) se logra identificar que el $4.7 \%$ de la población en México está dentro de esta tipo de familia Dinki, es importante recalcar que este autor lo identifica así como familia, cuyo interés para esta investigación es el estilo de vida; también puede encontrar que los del nido vacío pudieran entrar dentro de este segmento puesto que, son personas que tienen ingresos porque se encuentran en una etapa de jubilación, solo que para tal efectos este no está tomado en consideración. De igual forma Guillén (2016) "Se estima que en México los Dink representan del 5\% de los hogares, no obstante, su número aumentó considerablemente en los últimos años y se prevé que esta tendencia se mantenga".
Y la revista Merca 2.0 (2017), este tipo de familias mexicanas también representan el $4.7 \%$ con un Nivel Socioeconómico (NSE) bajo $\mathrm{D}+/ \mathrm{D}$.

Pintos (2012) establece siete categorías o tendencias sobre los Dinkis considerándolo como un target que persigue marcas Premium, dichas directrices son: Sin ataduras, Amantes de lo Premium, Vanguardia y Aventura, Veta Tech, Versión Dink de la Canasta Familiar, Cuando llega el Bebé y la Nueva Estructura.

Una de las ventajas comerciales es que este segmento de mercado, estilo de vida, nicho, target, tipo de familia, etc.; son personajes que culturalmente han generado un importante objetivo para las grandes corporaciones que los han observado y estudiado para su lanzamientos de productos o servicios.

Según datos estadísticos del Diario el Economista (2013) este tipo de parejas, que apenas rebasan el millón en México y considera que se ha vuelto una mina de oro para marcas, pues; sus hábitos de consumo están dirigidos a la demanda de bienes y servicios en una débil economía mexicana.

El mercado de los Double income no kids (parejas que recibe cada uno su salario y no tienen hijos) asiende a más de los 4,000 millones de dólares en México, según resultados de la consultora De la Riva Investigación Estratégica, cifra nada despreciable para aquellos empresarios que desean coloar un negocio, cumpliendo necesidades del nicho (Vargas, 2010).

Puesto que son un estilo de vida que no están bajo la presión del gasto de un hijo, suele poseer todas sus energías para concentrarse en compras de toda índole, así como la oportunidad de elegir destinos turísticos que sean de su agrado sin importar la cantidades exorbitantes que pudiesen gastar en esos gustos y deseos, lo que da pie a la alimentación de sus ego a través de sus dos factores sobresalientes como lo es el individualismo y el hedonismo para obtener lo que les complazca. 
Estas afirmaciones que muestran por un lado Orejuela (2017) sobre jóvenes acostumbrados a tener todo de forma rápida $\mathrm{y}$ fácil para ser felices lo que observo que la parte hedonista es la presencia del dinki, y por otro, la aportación de Castañeda (2014) con su revolución de efectos hacia una nueva estirpe social que ve el renunciar a no tener hijos como una oportunidad para generar una cultura de comodidad y sin compromisos.

Los dinkis "se caracterizan por su independencia, individualismo, narcisismo, despreocupación, dinamismo, espontaneidad y hendonismo (bienestar físico y psíquico" (Olamendi, 2008). Frente a esta situación Olamendi (2008) afirma que las mujeres tienden a tener una posición de igualdad frente al hombre.

Según Orejuela (2017) psicóloga afirma que "la sociedad actual promueve mucho el hedonismo, inmediatismo y mínimo esfuerzo. Jóvenes acostumbrados a tenerlo todo de forma fácil y rápida, sin límites, ni obligaciones, con la falsa idea de que lo merecen todo y que lo único importante es que ellos sean felices".

El crecimiento personal no esta tan alejado de las necesidades que buscan los Dinkis, puesto que en el surgimiento de lograr tener más ingreso y tener ese poder adquisitivo que desean, están inmersos en el mundo de los libros, pues se la pasan preparándose académicamente estudiando más con el fin de poder escalonar a cargos y puestos de trabajo que les proporcione ese estatus económico adhoc que persigue y cubrir sus necesidades.

Se considera que "sus simpatizantes son jóvenes y suelen tener un buen trabajo. Sobre todo, se definen como "neoluditas": no están dispuestos a renunciar a su tiempo para cargar con el enorme peso que supone alimentar, cuidar, educar y atender a un hijo" (Castañeda, 2014). Se supone que "el modelo es atractivo: prolongan la juventud y disfrutan de todas las cosas que no serían totalmente posibles de comprometerse y fundar una familia" (Olamendi, 2008). Estas parejas suelen tener una estabilidad económica buena y que decir a nivel profesional, por lo que logran aprovechar los tiempos en un sinfín de actividades, sin importarles el futuro.
Este nuevo colectivo compra el doble de libros y música que las parejas tradicionales, viajan un $78 \%$ más que el resto de la población y eligen con más frecuencia lugares nuevos" (Mundo, 2005).

Para Goldberg citada en Amadeo (2013) explica que:

"Hay parejas que consensuan no tener hijos y ponen toda la libido en el consumo, los viajes y el desarrollo personal y profesional. A veces, comparten una actividad o pasión, como el cine. Prefieren tener su libertad plena y no estar atados a nada ni a nadie".

Pues para algunas empresas es un estilo de vida o nicho de mercado atractivo para generar ingresos, pues para algunas agencias de bienes raíces ha significado el esquema para crear conceptos habitacionales dirigidos a este tipo de estilo de vida, tal es el ejemplo en Escocia, en la pequeña villa Firhall.

La tal mencionada Firhall fue creada en el 2003, es una comunidad sólo para adultos para cualquier childfree o dinki que buscan vivir en un espacio de paz con elevada calidad de vida. Considerada como "el lugar donde viven los ogros que odian a los niños" (Child Free, 2017).

En Colima los datos y la importancia del crecimiento poblacional no se encuentra del todo alejado del interés de la sociedad, en una columna del Ecos de la Costa, Hernández Chavarín (2017) hace ya mención sobre dichos cambios en la disminución de los integrantes de la familia al abordar los siguiente:

"El INEGI expone que los hogares familiares pueden dividirse en clases, que se conforman a partir del tipo de parentesco que se tenga con el jefe del hogar, denominados nucleares, ampliados y compuestos. El primero lo conforma por el núcleo familiar de primera generación, es decir, parejas con hijos o parejas sin hijos..."

Con ello se puede abordar que en Colima al menos ya se empieza a notar preocupación por dicho tema relacionado a las parejas sin hijos. 
De igual forma en el Decreto No. 559 por el que se aprueba la Ley que regula los derechos de las jefas de familia del Estado de Colima con fecha del 10 de Octubre de 2010, dicha iniciativa prevé un padrón estatal con una clasificación de los perfiles estando dentro de ellas la clasificación de: Casadas sin hijos...

Con todo esto, se ha despertado la inquietud por su estudio para conocerlos y describirlos, pues solo se han encontrado datos de quienes son, cuanto gastan anualmente, en que invierte su tiempo y dinero, así como los tipos de productos, marcas y servicios que son disponibles para ellos, por lo cual nacen interrogantes, como ¿Qué hacen para acercarlos a ellos? ¿Cómo buscan los lugares que van dirigidos a ellos? ¿En dónde se encuentran los dinkis en el estado? ¿Existen lugares en el Estado que sean exclusivo para ellos? ¿A qué lugares acuden? Entre otras.

Aunque se sabe que no es un segmento o estilo de vida tan cercano a estos tiempos, si es de suma importancia hacer mención que su descubrimiento no ha sido circunstancial, ni mucho menos una consecuencia al crecimiento poblacional, sino que este estilo de vida es por elección y convencimiento por parte de quienes lo ejercen.

Sin embargo, de acuerdo con las condiciones que se presenta la investigación, se ha detectado que no existe una oferta fundamentada que haga énfasis sobre el uso, adquisición, disposición y disfrute de todos los servicios y productos que los Dinkis compran, y que este ofrecimiento conlleve todos los beneficios que el estilo de vida necesita.

Por lo que se considera que sea una herramienta útil como una oferta con personalización con la premisa de que se tienen diferentes gustos por lo que su experiencia en el manejo interactivo que les permita descubrir sus lugares, productos y servicios de su preferencia. Estableciendose la interrogante $¿$ Existen este estilo de vida hedonista y utilitarista en las parejas Dinkis en los municipios de Colima y Villa de Àlvarez?

\section{Metodología a desarrollar}

Para efectos de la investigación fue necesario establecer un tipo de Investigación Cuantitativo, pues se pretende encontrar los rasgos del fenómeno estudiado y conocer la relación entre los hábitos de consumo hedonista y utilitarista, así como las características que presenta este estilo de vida (parejas sin hijos). A través de las variables estudiadas se platearon las siguientes hipótesis:

H1: Se cree que el estilo de vida hedonista y Utilitarista está presente en las parejas Dinkis en los municipios de Colima y Villa de Álvarez.

H0: Se cree que el estilo de vida hedonista y utilitarista no está presente en las parejas Dinkis en los municipios de Colima y Villa de Álvarez. Con ello el modelo hipótetico se manifiesta en el siguiente constructo (ver Figura 1).

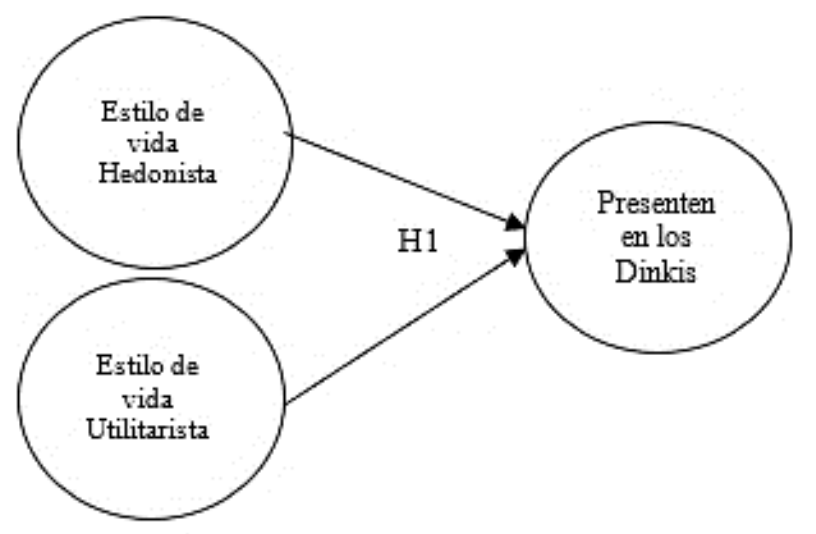

Figura 1 Modelo hipótetico- constructo

Este análisis investigativo de los Dink's es de enfoque exploratorio, pues lo primero es encontrar y conocer si existe el estilo de vida en los Municipios Colima y Villa de Álvarez y descriptiva, se requiere describir los aspectos que presenta este estilo de vida y el grado hedonista o utilitarista que presentan ambos géneros en el estado de Colima, dentro de los Municipio de Colima y Villa de Álvarez.

La investigación descriptiva se soporta principalmente en técnicas como la encuesta, la entrevista, la observación y la revisión documental. (Bernal, 2010, pág. 113), por lo que las técnicas que se utilizaron para efectos de esta investigación son: LA ENCUESTA. 
El instrumento que se llevará acabo pretende medir las dos variables que permita describir el estilo de vida de los Dinkis y el grado de hedonismo y utilitarista que presentan los Dinkis. Con base a Brata \& Ahtola (1991) se medirá el hedonismo y utilitarismo la cual esta estructura bajo la escala de diferencial semántica, el instrumento esta estructurado con 4 adjetivos opuestos en cada extremo para medir el hedonismo y otros 4 adjetivos para el utilitarismo, además de que en la escala se pretende evaluar su pensar con relación a la compra de los artícuos o servicios como automóvil, calzado, ropa, viajes, alimentos, vivienda, tecnología.

La muestra se determinó del universo entre los municipios de Villa de Alvarez y Colima siendo parejas casadas con edad entre 25 y 40 años como criterio de inclusión, con una muestra no probabilística, con el tipo de muestreo conveniencia y para la recolección de datos se hizo de manera electrónica con Google formulario durantes los meses de septiembre 2018 a marzo 2019 a través de bola de nieve obteniendo 224 participantes de los cuales el $50.9 \% \quad(n=114)$ eran hombres y el $49.1 \%$ $(n=110)$ mujeres, la edad promedio que más contestaron son de 25 años $24.1 \%(n=54)$, de 34 años $11.6 \%(n=26)$ y de 32 años $8.9 \%(n=20)$, siendo el nivel educativo de licenciatura el que predomino con $63.4 \%(n=142)$, seguido de la maestría $27.7 \%(n=62)$, y la localidad donde más contestaron fueron del municipio de Colima con $62.5 \%(n=140)$ y Villa de Álvarez 37.5\% $(n=84)$ y posterior a ello se hizo la validación de las variables para medir su consistencia interna con el Alfa de Cronbach (ver Tabla 1).

El tratamiento que se hizo a los resultados en primera instancia se trabajo un análisis decriptivo para conocer su datos de varianza, media y desviación, para posterior a ello establecer una analisis de componentes principales (ACP) con el objetivo de reducción de los elementos para establecer grupos de analísis, además de ello establecer regresión lineal para comprobar la hipótesis planteada en la investigación.

\begin{tabular}{|lr|l|}
\hline \multicolumn{3}{|c|}{ Reliability Statistics } \\
\hline Cronbach's Alpha & N of Items \\
\hline & .932 & \\
\hline
\end{tabular}

Tabla 1 Alfa de Cronbach

\section{Resultados}

Con base a los resultados de la encuesta aplicado a 224 Dinkis se tienen los siguientes hallazgos:

La encuesta estuvo dividida en dos bloques, Preguntas filtros, así como la medición del Hedonismo y Utilitarismo. De los encuestados y con relación a las preguntas filtros establecidas el $100 \%$ cumple la característica, vive con su pareja no tienen hijos y ambos trabajan, siendo el sector público $57.1 \%(\mathrm{n}=128)$ en el que se desarrollan laboralmente seguido del privado e independiente lo que indica que son Dinkis.

Para el caso de saber si los dink's llevan un estilo de vida hedonista o utilitarista, se presenta los siguientes resultados:

Mediante la prueba de esfericidad de Bartlett, se asegura que si el nivel crítico es superior a 0.05 entonces no se rechaza el supuesto nulo de esfericidad, pero como se observa al comprobar este análisis la significancia es perfecta, ya que se obtiene el valor 0,000 , por lo tanto, se puede decir que el análisis factorial es factible.

Y la prueba KMO muestra que la relación entre las variables es baja con .465 alejado de 1, es por lo que el análisis factorial según la literatura no se debe utilizar un análisis factorial. Con estos datos de KMO baja, se procedio a hacer una transformación de las variables y posterior a ello se volvio a correo el analisis de reducción de dimensión obteniendo los siguientes datos (Tabla 2) del KMO y Bartlett ahora ya con una relación de .664 y una significancia de .000 con ello se puede predecir ya un analisis factorial.

El p-valor $=0$ con lo que existe correlación significativa entre las variables.

\begin{tabular}{|c|c|c|}
\hline \multicolumn{3}{|c|}{ KMO y prueba de Bartlett } \\
\hline \multicolumn{2}{|c|}{$\begin{array}{l}\text { Medida de adecuación muestral de Kaiser- } \\
\text { Meyer-Olkin. }\end{array}$} & .664 \\
\hline \multirow[t]{3}{*}{$\begin{array}{l}\text { Prueba de esfericidad } \\
\text { de Bartlett }\end{array}$} & $\begin{array}{l}\text { Chi-cuadrado } \\
\text { aproximado }\end{array}$ & 1074.362 \\
\hline & $\mathrm{gl}$ & 45 \\
\hline & Sig. & .000 \\
\hline
\end{tabular}

Tabla 2 KMO y prueba de Bartlett Utilitarismo y Hedonismos 
En las comunalidades se expresa la parte de las variabilidades que debe teener cada variable explicada por grupos los resutlados su extracción fueron altas en donde el valor inicial era 1, siendo uno dato el que se encuetra por debajo del 0.5, el U Viajar el cual muestra una extracción de .484 como se puede observar en la tabla 3.

\begin{tabular}{|l|r|r|}
\hline \multicolumn{3}{|c|}{ Comunalidades } \\
\hline & Inicial & Extracción \\
\hline U Compra.auto & 1.000 & .768 \\
\hline H Compra Auto & 1.000 & .814 \\
\hline H Compra apar.eléctrico & 1.000 & .701 \\
\hline U Compra apar.eléctrico & 1.000 & .696 \\
\hline U Calzado y Ropa & 1.000 & .675 \\
\hline H Calzado y Ropa & 1.000 & .642 \\
\hline U Viajar & 1.000 & .626 \\
\hline H Viajar & 1.000 & .484 \\
\hline U Alimentos & 1.000 & .809 \\
\hline H Alimentos & 1.000 & .707 \\
\hline $\begin{array}{l}\text { Método de extracción: Análisis de Componentes } \\
\text { Principales. }\end{array}$ \\
\hline
\end{tabular}

Tabla 3 Comunalidades Hedonismo y Utilitarismo

En la tabla de varianza total explicada se observa que $69.214 \%$ se encuentrá entre los compentes 1,2 y 3 , correspondiendo en el componente 1 un $41.658 \%$ de la varianza total, el $15.990 \%$ para el componente 2 y $11.566 \%$ el componente 3 , el resto de la varianza total se explica con los 6 componentes restantes, con porcentajes mejores a $10 \%$ (Tabla 4$)$.

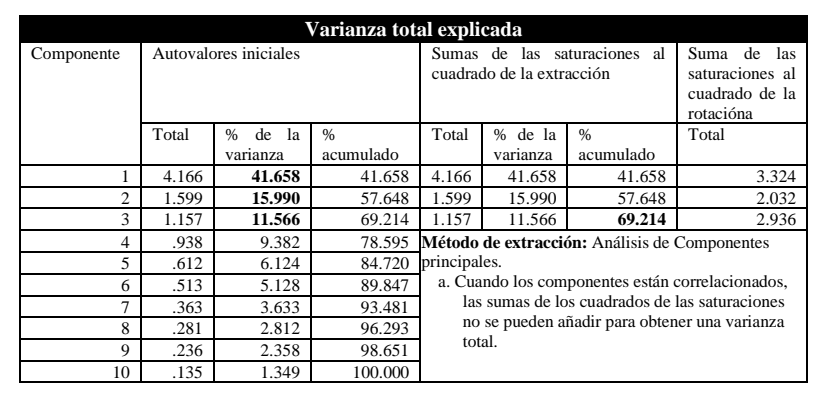

Tabla 4 Varianza total explicada Hedonismo y Utilitarismo

La matriz de componentes (ver Tabla 5) permitió analizar cuales de los elementos estan cargando en cada unos de los componentes extraidos. Como se puede observar surgen 3 componentes, en el componente carga 5 categorías del Hedonismo (Compra apareléctico, Alimentos, Calzado y Ropa, Viajar y Compra Auto) y 3 categorías del Utilitarismo (Calzado y Ropa, Compra apar.eléctrico y Alimentos).

\begin{tabular}{|c|c|c|c|}
\hline \multicolumn{4}{|c|}{ Matriz de componentes ${ }^{a}$} \\
\hline & \multicolumn{3}{|c|}{ Componente } \\
\hline & 1 & 2 & 3 \\
\hline H Compra apar.eléctrico & 754 & 240 & -.273 \\
\hline U Calzado y Ropa & .712 & -.360 & -.196 \\
\hline U Compra apar.eléctrico & .691 & -.250 & -.395 \\
\hline U Alimentos & .660 & & .605 \\
\hline H Alimentos & .657 & -.208 & .481 \\
\hline H Calzado y Ropa & .648 & & -.470 \\
\hline H Viajar & .642 & -.223 & .146 \\
\hline H Compra Auto & .635 & .615 & .180 \\
\hline U Compra.auto & .507 & .709 & \\
\hline U Viajar & .503 & -.605 & \\
\hline \multicolumn{4}{|c|}{$\begin{array}{l}\text { Método de extracción: Análisis de componentes } \\
\text { principales. }\end{array}$} \\
\hline a. 3 componentes extraíc & & & \\
\hline
\end{tabular}

Tabla 5 Matriz de componentes del Hedonismo y Utilitarismo

Entonces se puede argumentar, que a las parejas Dinkis; el placer por realizar compras esta latente con mayor presencia en los aparatos eléctricos, de igual forma la parte de utilitarismo en la compra de calzado y ropa. El componente 2 esta de igual forma la presencia del Hedonismo con la Compra Auto y 2 categorías del Utilitarismo (Compra auto, viajar el cual carga inversamente). Una vez establecido el analisis de reducción de dimensiones, se realizó un analisis de regresión para determinar la relación entre las variables, para este caso la variable dependiente se tomo Motivos de Compra (necesidad, impulso, vanguardia, constumbre, otros) y las variables independientes Hedonismo y Utilitarismo (compra automovil, compra apar.eléctrico, calzado y ropa, viajar y alimentos). Los datos se transformaron para un mejor analisis quedando resultados como los siguientes:

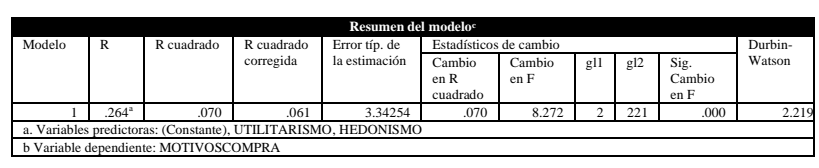

Tabla 6 Resumen del Modelo Hedonismo y Utilitarismo

Las variables independientes incluidas en el analisis explican el $6.1 \%$ de la variable dependiente pues R 2 corregida $=0.061$, además, el error típico de los residuos (3.34254 en el análisis de regresión múltiple), lo que indica una mejora en el ajuste, pues el coeficiente de $\mathrm{R}$ denota que existe una correlación media de .264 entre la varibale dependiente (MOTIVOSCOMPRA) y las variables independientes (Hedonismo y Utilitarismo). 
En el puntaje de la prueba de DurbinWatson indica que existe una correlación de 2.219 , dado el supuesto que si el valor esta en 1 y 3 se acepta la independencia. El estadístico F (resumen de ANOVA) es el que contrasta la hipótesis nula de que el valor poblacional de $\mathrm{R}$ es cero, como $\mathrm{p}=.000<.005$, por tanto, permite decidir si existe relación lineal significativa entre la variable "MOTIVOSCOMPRA" y las variables "Utilitarismo y Hedonismo" tomadas juntas. El valor del nivel crítico con Sig. $=0,000$ indica que sí existe relación lineal significativa, por lo que las variables poseen un buen ajuste, por que se puede recharzar la hipótesis nula.

\begin{tabular}{|c|c|c|c|c|c|c|}
\hline \multicolumn{7}{|c|}{ ANOVA $^{a}$} \\
\hline \multicolumn{2}{|c|}{ Modelo } & Suma de & $\mathrm{gl}$ & Media & $\mathrm{F}$ & Sig. \\
\hline \multirow[t]{3}{*}{1} & Regresión & 184.845 & 2 & 92.422 & 8.272 & $.000^{\mathrm{b}}$ \\
\hline & Residual & 2469.138 & 221 & 11.173 & & \\
\hline & Total & 2653.982 & 223 & & & \\
\hline \multicolumn{7}{|c|}{ a. Variable dependiente: MOTIVOSCOMPRA } \\
\hline \multicolumn{7}{|c|}{$\begin{array}{l}\text { b. Variables predictoras: (Constante), UTILITARISMO, } \\
\text { HEDONISMO }\end{array}$} \\
\hline
\end{tabular}

Tabla 7 Anova del Hedonismo y Utilitarismo

Para la construcción del modelo se necesita la constante y un coeficiente, para efecto se necesita la fórmula $\mathrm{y}=\mathrm{a}+\mathrm{bx}$, por lo tanto sustituyendo queda de la siguiente forma:

\section{UTILITARISMO.}

$$
\mathrm{Y}=11.101-.091 \text { HEDONISMO }+.082
$$

El coeficiente correspondiente a la contante que es el origen de la recta de regresión y el coeficiente correspondiente a las variables independientes (hedonismo y utilitarismo) es la pendiente de la recta, lo que nos muestra un cambio en la variable dependiente (motivocompra) por cada cambio de las variables independientes mencionadas. En las salidas se puede observar que las variables predictoras, son significativas ya que sus p-valor son mayores a la del nivel de alfa común 0.05 , con Sig. 0.000, el resto.

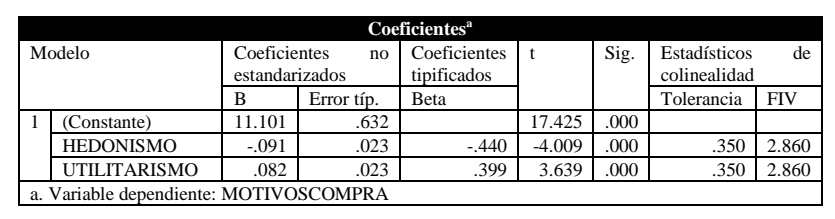

Tabla 8 Coeficientes de Hedonismo y Utilitarismo
Además de acuerdo con el coeficiente tificados o estandarizados la variable Hedonismo es la que más aporta al modelo con un $44.0 \%$ de forma inversa con respecto al valor de Beta -.440 , y la variable Utilitarismo afecta con un $39.9 \%$ con base a Beta .399, quedando la ecuación de regresión no estandarizada como:

$\mathrm{Y}=-.440$ Hedonismo +.399 Utilitarismo.

De acuerdo con las pruebas de significación, sirven para contrastar la hipótesis nula de que un coeficiente de regresión vale cero en la población. Si niveles críticos (Sig.) pequeños $(<0.05)$ indica que se debe rechazar la hipótesis nula. Por lo que ambas variables Hedonismo y Utilitarismo tienen significancia estadística de .000 . De acuerdo al grado de confianza de $.95 \%$, por lo tanto el nivel de significancia permite no rechar la hipótesis nula.

H1 Se cree que el estilo de vida hedonista e individualista está presente en las parejas Dinkis en los municipios de Colima y Villa de Álvarez.

Ho Se cree que el estilo de vida hedonista e individualista no está presente en las parejas Dinkis en los municipios de Colima y Villa de Álvarez.

\section{Agradecimiento}

Se hace un merecido agradecimiento a mi institución que me ha brindado los acercamientos a la investigación además a la Dra. María de los Dolores Santarriaga Pineda, que ha brindado su conocimiento para la conformación profesional de su servidor quedando eternamente agradecido, de igual forma a todos los que hicieron posible que este artículo fuera posible.

\section{Conclusiones}

Con base a los resultados obtenidos y a través de ellos se puede decir que las hipótesis planteadas y con la sufiente carga estadística presentada se puede decir que los motivos de compra por parte de los Dinkis tiene una relación con las dos variables presentadas el hedonismo y utilitarismo ya que influyen significativamente con valores del supuesto que si el nivel de significancia es $>.005$ la hipótesis nula se acepta y si es <.005 la hipótesis nula se rechaza.

SÁNCHEZ-FIGUEROA, Cruz Osbaldo. Las características de los Dinki's: factores de hedonismo y utilitarismo influyentes en el comportamiento de consumo. Revista de Sociología Contemporánea. 2019 
Con ello encontramos que la para efectos de esta investigación el hedonismo y el utilitarismo no esta presente en las parejas Dinkis de los municipios de Colima y Villa de Álvarez, con niveles de Sig. .000 en Anova.

En ese tenor, bajo el modelo propuesto en la investigación se puede observar que el hedonismo presentado en los Dinkis deriva en algunas acciones como lo son los autos, viajar y alimentos, pero por otro lado, el utilitarismo esta presente en la compra de ropa y calzado, así como, en viajar, compra de auto o aparatos electrónicos.

Escenarios no presentes en los municipios de Colima y Villa de Álvarez, al indicar que son jóvenes que promueven el hedonismo, inmediatismo y mínimo esfuerzo (Orejuela A., 2017). Puesto que al comporbar que no generan una condición relacionada con el comportamiento de compra (motivos) que lo hagan por cuestiones de placer o de utilidad.

\section{Referencias}

Amadeo, E. (1 de agosto de 2013). Infobae. Recuperado el 17 de julio de 2018, de Infobae: https://www.infobae.com/2013/08/01/722898la-vida-child-free/

Bernal, C. A. (2010). Metodología de la Investigación (TERCERA ed.). Colombia: Pearson Educación.

Castañeda B., J. (2014). Patologías Urbanas Ecografía de una socieda desestructurada. UOC, S.L.

Child Free. (1 de Agosto de 2017). Child Free. Recuperado el 12 de julio de 2018, de Child Free: http://childfree.com.mx/conoce-firhallpueblo-sin-ninos/

Guillén, G. (26 de Agosto de 2016). ActualMX.com. Recuperado el 02 de Junio de 2018, de ActualMX.com: http://www.actualmx.com/parejas-dink-nuevomodelo-familiar/

Hernández Chavarín, E. (09 de Marzo de 2017). Cuestión de Enfoque: ¿Y la familia en Colima? Ecos de la Costa.

López Romo, H. (Octubre de 2016). Los Once tipos de familias en México. Revista Digital DDT(47), 38.
Mundo, E. (18 de NOVIEMBRE de 2005). EL MUNDO. pág. 1. Obtenido de http://www.elmundo.es/elmundo/2005/11/18/so ciedad/1132312243.html

Olamendi, G. (27 de MAYO de 2008). MARKTING RACIONAL. Obtenido de http://mkrelacional.blogspot.com/2008/05/losdinkis.html.

Orejuela A., E. (17 de Julio de 2017). Revista Vive. Obtenido de Revista Vive: http://revistavive.com/parejas-dinkis/

Vargas, I. (19 de ENERO de 2010). PAREJAS SIN HIJOS, CLIENTES CON DINERO. EXPANSIÓN. Recuperado el 2017, de $\mathrm{http} / / /$ expansion.mx/mi-

dinero/2010/01/18/parejas-sin-hijos-clientescon-dinero 